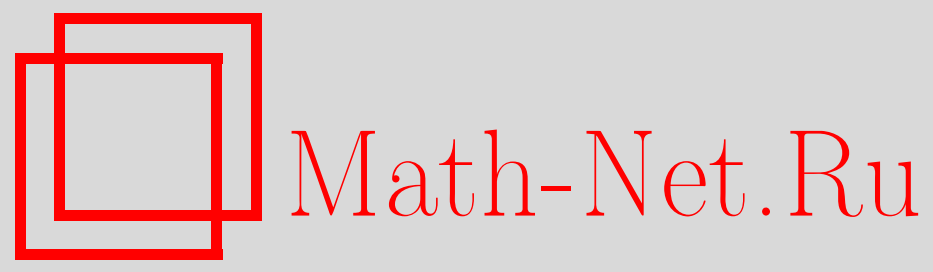

М. М. Глухов, Инъективные отображения слов, не размножающие искажений типа пропусков букв, Дискрет. матем., 1999, том 11, выпуск 2, 20-39

DOI: https://doi.org/10.4213/dm371

Использование Общероссийского математического портала Math-Net.Ru подразумевает, что вы прочитали и согласны с пользовательским соглашением http: //www . mathnet.ru/rus/agreement

Параметры загрузки:

IP: 34.229 .45 .116

26 апреля 2023 г., 13:20:03 


\title{
Инъективные отображения слов, не размножающие искажений типа пропусков букв
}

(C) 1999 г. М. М. Глухов

\begin{abstract}
Пусть $A^{*}$ - множество всех слов конечных длин в алфавите $A$. Дается полное описание всех таких инъективных отображений множества $\Omega^{*}$ в множество $\Omega_{1}^{*}$, которые не размножают ошибки типа пропуска букв. Предполагается, что алфавиты $\Omega$ и $\Omega_{1}$ конечны.
\end{abstract}

Данная работа является продолжением статьи [1]. В связи с этим мы будем пользоваться обозначениями из [1]. Если $\Omega$ - некоторый конечный алфавит, то $\Omega^{t}-$ множество слов длины $t, \Omega^{*}-$ множество всех слов в алфавите $\Omega, \Lambda$ - пустое слово, $d(P)$ - длина слова $P, P=G$ - графическое равенство слов, $P^{(i)}-i$-я буква слова $P, \mu(P)$ - слово $a_{n} a_{n-1} \ldots a_{1}$ для слова $P=a_{1} \ldots a_{n-1} a_{n}, \varepsilon-$ бинарное отношение, для которого $P \varepsilon Q$ означает, что слово $Q$ получено из $P$ удалением одного вхождения какой-либо одной буквы, $G(\Omega, \varepsilon)$ - множество всех инъективных отображений $\varphi: \Omega^{*} \rightarrow \Omega^{*}$, не размножающих искажений типа пропуска букв в словах, т. е. удовлетворяющих условијо

$$
\forall P, Q \in \Omega^{*}, \forall i \in\{1, \ldots, d(P)\}, \exists j \in\{1, \ldots, i\}\left(P \varepsilon^{i} Q \Rightarrow \varphi(P) \varepsilon^{j} \varphi(Q)\right),
$$

$G_{1}(\Omega, \varepsilon)$ - множество всех биективных отображений из $G(\Omega, \varepsilon)$. Будут использоваться также общепринятые обозначения: $\mathbf{N}-$ множество натуральных чисел, $\mathbf{N}_{0}=$ $\mathbf{N} \cup\{0\}$ и другие.

Один из основных результатов работы [1] заключался в описании всех отображений из $G_{1}(\Omega, \varepsilon)$. Оказалось, что $G_{1}(\Omega, \varepsilon)$ состоит из отображений $\varphi$ видов

$$
\forall P \in \Omega^{*}: \varphi(P)=P^{\sigma}
$$

и

$$
\forall P \in \Omega^{*}: \varphi(P)=\mu(P)^{\sigma},
$$

где $\sigma$ - любая фиксированная для данного $\varphi$ подстановка множества $\Omega$. Однако вопрос описания отображений из $G(\Omega, \varepsilon)$, также представляющий практический интерес, остался открытым. И тем более не рассматривался в [1] вопрос описания множества $G\left(\Omega, \Omega_{1}, \varepsilon\right)$ всех отображений $\varphi: \Omega^{*} \rightarrow \Omega_{1}^{*}$, удовлетворяющих условию (1), при различных алфавитах $\Omega, \Omega_{1}$. Решению этого общего вопроса и посвящена 
данная работа. В рассматриваемом случае, как и в [1], доказывается, что условие (1) равносильно условию

$$
\forall P, Q \in \Omega^{*}(P \varepsilon Q \Rightarrow \varphi(P) \varepsilon \varphi(Q)),
$$

и что любое отображение $\varphi$ из $G\left(\Omega, \Omega_{1}, \varepsilon\right)$ переводит $\Omega^{t}$ в $\Omega_{1}^{t+\Delta}$ при любом $t \in \mathbf{N}_{0}$ и подходящем, фиксированном для заданного $\varphi$, значении $\Delta \in \mathbf{N}_{0}$. В отличие от рассмотренного в [1] случая здесь особо выделяется подслучай, в котором $|\Omega|=2$.

Для формулировки результатов введем еще обозначение $I\left(\Omega, \Omega_{1}\right)$ для множества всех инъективных отображений $\varphi: \Omega \rightarrow \Omega_{1}$. По аналогии с [1] через $P^{\sigma}$ для $\sigma \in$ $I\left(\Omega, \Omega_{1}\right)$ будет обозначаться слово, полученное из $P$ заменой всех букв их образами при отображении $\sigma$.

Основные результаты данной работы можно сформулировать в виде двух теорем.

Теорема 1. Если $|\Omega| \geqslant 3 u\left|\Omega_{1}\right| \geqslant|\Omega|$, то множество $G\left(\Omega, \Omega_{1}, \varepsilon\right)$ coстоит из всех отображений, задаваемых формулами

$$
\forall P \in \Omega^{*}: \varphi(P)=A P^{\sigma} B
$$

$u$

$$
\forall P \in \Omega^{*}: \varphi(P)=A \mu(P)^{\sigma} B
$$

при произволъных, фиксированных для каждого $\varphi$, словах $A, B \in \Omega_{1}^{*}$ и отображениях $\sigma \in I\left(\Omega, \Omega_{1}\right)$.

Теорема 2. Если $\Omega=\{a, b\}$ u $\left|\Omega_{1}\right| \geqslant|\Omega|$, то множество $G\left(\Omega, \Omega_{1}, \varepsilon\right)$ состоит из всех отображений $\psi$, задаваемых формулами

$$
\forall P \in \Omega^{*}: \psi(P)=A \varphi_{s, t, u, v, \sigma, \tau}(P) B
$$

$u$

$$
\forall P \in \Omega^{*}: \psi(P)=A \varphi_{s, t, u, v, \sigma, \tau}(\mu(P)) B
$$

при фиксированнъх для каждого $\psi$ параметрах

$$
A, B \in \Omega_{1}^{*}, \quad s, t \in \mathbf{N}_{0}, \quad u, v \in\{0,1\}, \quad \sigma, \tau \in I\left(\Omega, \Omega_{1}\right),
$$

по которым отображение $\varphi_{s, t, u, v, \sigma, \tau}: \Omega^{*} \rightarrow \Omega_{1}^{*}$ определяется следующим образом:

$$
\begin{aligned}
\forall n \in \mathbf{N}, \forall P \in \Omega^{n}: \varphi_{s, t, u, v, \sigma, \tau}(P) & =\left(\left(\bar{P}^{(1)}\right)^{u}\left(P^{(1)} \bar{P}^{(1)}\right)^{s} P\left(\bar{P}^{(n)} P^{(n)}\right)^{t}\left(\bar{P}^{(n)}\right)^{v}\right)^{\sigma} \\
\varphi_{s, t, u, v, \sigma, \tau}(\Lambda) & =\left(a^{u}(b a)^{s+t} b^{v}\right)^{\tau} .
\end{aligned}
$$

Здесъ $и$ ниже для любой буквы $x \in \Omega$ под $\bar{x}, x^{1}, x^{0}$ следует понимать соответственно другую букву из $\Omega$, саму букву $x$ и пустое слово $\Lambda$.

Для доказательства теорем 1, 2 нам понадобится ряд лемм. Сначала сформулируем две очевидные леммы об общих свойствах слов и бинарного отношения $\varepsilon$.

Лемма 1. Пусть $x_{1}, x_{2}, y_{1}, y_{2} \in \Omega, P \in \Omega^{*}$. Тогда

(a) если $x_{1} P=P y_{1}$, mо $x_{1}=y_{1} u P=x_{1}^{k}$ для некоторого $k \in \mathbf{N}_{0}$;

(b) если $x_{1} x_{2} P=P y_{1} y_{2}$, то или $P=\left(x_{1} x_{2}\right)^{k}$ для некоторого $k \in \mathbf{N}_{0} u x_{1}=y_{1}$, $x_{2}=y_{2}$, или $P=\left(x_{1} x_{2}\right)^{k} x_{1}$ для некоторого $k \in \mathbf{N}_{0} u x_{1}=y_{2}, x_{2}=y_{1}$. 
Лемма 2. Пусть $x, y \in \Omega, x \neq y, t \in \mathbf{N}_{0}, n \in\{0,1\}, A, B \in \Omega^{*} u X$ - неизвестное слово из $\Omega^{*}$. Тогда

(а) система уравнений

$$
\begin{aligned}
& A(x y)^{t} x^{n} B \varepsilon X \\
& A(y x)^{t} y^{n} B \varepsilon X
\end{aligned}
$$

имеет ровно два реиения

$$
\begin{aligned}
& X_{1}=A y(x y)^{t-1} x^{n} B, \\
& X_{2}=A x(y x)^{t-1} y^{n} B
\end{aligned}
$$

при $t>0$ и ровно одно решение $X=A B$ при $t=0, n=1$;

(b) система уравнений

$$
\begin{aligned}
& X \varepsilon A(x y)^{t} x^{n} B, \\
& X \varepsilon A(y x)^{t} y^{n} B
\end{aligned}
$$

имеет ровно два речиения

$$
\begin{aligned}
& X_{1}=A y(x y)^{t} x^{n} B \\
& X_{2}=A x(y x)^{t} y^{n} B
\end{aligned}
$$

$n p u t+n>0$.

Утверждения леммы 1 доказываются путем побуквенного сравнения слов из заданных равенств. Утверждения леммы 2 доказываются путем рассмотрения возможных вариантов удаления (вставки) букв в исходные слова при переходе от них к слову $X$. По существу, с учетом симметрии дело сводится к одному случаю, когда в первом слове производится удаление (вставка) в подслове $A$, а во втором в подслове $B$.

Лемма 3. Если $\varphi \in G(\Omega, \varepsilon), a, b \in \Omega u a \neq b$, то выполняется одно из следующих шести условии:

$\left(1^{\circ}\right) \varphi(a)=A b_{1} B, \varphi(b)=A b_{2} B$,

$\varphi(a b)=A b_{1} b_{2} B, \varphi(b a)=A b_{2} b_{1} B ;$

$\left(2^{\circ}\right) \varphi(a)=A b_{1} B, \varphi(b)=A b_{2} B$,

$\varphi(a b)=A b_{2} b_{1} B, \varphi(b a)=A b_{1} b_{2} B ;$

$\left(3^{\circ}\right) \varphi(a)=A b_{1}\left(b_{2} b_{1}\right)^{r} B, \varphi(b)=A b_{2}\left(b_{1} b_{2}\right)^{r} B, r \in \mathbf{N}$,

$\varphi(a b)=A\left(b_{1} b_{2}\right)^{r+1} B, \varphi(b a)=A\left(b_{2} b_{1}\right)^{r+1} B ;$

$\left(4^{\circ}\right) \varphi(a)=A b_{1}\left(b_{2} b_{1}\right)^{r} B, \varphi(b)=A b_{2}\left(b_{1} b_{2}\right)^{r} B, r \in \mathbf{N}$,

$\varphi(a b)=A\left(b_{2} b_{1}\right)^{r+1} B, \varphi(b a)=A\left(b_{1} b_{2}\right)^{r+1} B ;$ 
$\left(5^{\circ}\right) \varphi(a)=A\left(b_{1} b_{2}\right)^{r} B, \varphi(b)=A\left(b_{2} b_{1}\right)^{r} B, r \in \mathbf{N}$,

$$
\varphi(a b)=A b_{2}\left(b_{1} b_{2}\right)^{r} B, \varphi(b a)=A b_{1}\left(b_{2} b_{1}\right)^{r} B ;
$$

$\left(6^{\circ}\right) \varphi(a)=A\left(b_{1} b_{2}\right)^{r} B, \varphi(b)=A\left(b_{2} b_{1}\right)^{r} B, r \in \mathbf{N}$,

$$
\varphi(a b)=A b_{1}\left(b_{2} b_{1}\right)^{r} B, \varphi(b a)=A b_{2}\left(b_{1} b_{2}\right)^{r} B,
$$

где $A, B$ - некоторие слова из $\Omega_{1}^{*}, b_{1}, b_{2} \in \Omega_{1} u b_{1} \neq b_{2}$.

Доказательство. Пусть $\varphi(\Lambda)=C$. Так как $a \varepsilon \Lambda, b \varepsilon \Lambda$, то $\varphi(a) \varepsilon C, \varphi(b) \varepsilon C$, и потому

$$
\varphi(a)=P_{1} c P_{2}, \quad \varphi(b)=P_{1}^{\prime} d P_{2}^{\prime},
$$

где $P_{1} P_{2}=P_{1}^{\prime} P_{2}^{\prime}=C$.

Рассмотрим слова $Q_{1}=\varphi(a b), Q_{2}=\varphi(b a)$. Так как $a b \varepsilon a, b a \varepsilon a, a b \varepsilon b, b a \varepsilon b$, должны выполняться соотношения

$$
Q_{i} \varepsilon P_{1} c P_{2}, \quad Q_{i} \varepsilon P_{1}^{\prime} d P_{2}^{\prime}, \quad i=1,2 .
$$

Тогда слова $Q_{1}, Q_{2}$ должны иметь вид

$$
\begin{aligned}
& Q_{1}=A_{1} b_{1} A_{2} c_{1} A_{3}, \\
& Q_{2}=B_{1} b_{2} B_{2} c_{2} B_{3}
\end{aligned}
$$

и априори для слов $P_{1} c P_{2}, P_{1}^{\prime} d P_{2}^{\prime}$ возможны лишь следующие четыре варианта.

Вариант I:

$$
\begin{aligned}
& P_{1} c P_{2}=A_{1} A_{2} c_{1} A_{3}=B_{1} B_{2} c_{2} B_{3} \\
& P_{1}^{\prime} d P_{2}^{\prime}=A_{1} b_{1} A_{2} A_{3}=B_{1} b_{2} B_{2} B_{3}
\end{aligned}
$$

Вариант II:

$$
\begin{aligned}
& P_{1} c P_{2}=A_{1} A_{2} c_{1} A_{3}=B_{1} b_{2} B_{2} B_{3} \\
& P_{1}^{\prime} d P_{2}^{\prime}=A_{1} b_{1} A_{2} A_{3}=B_{1} B_{2} c_{2} B_{3} .
\end{aligned}
$$

Варианты III, IV получаются из I, II заменой $P_{1} c P_{2}$ на $P_{1}^{\prime} d P_{2}^{\prime}$ и $P_{1}^{\prime} d P_{2}^{\prime}$ на $P_{1} c P_{2}$ в левых частях соотношений (2)-(5).

Рассмотрим эти варианты.

Вариант I. Заметим сначала, что если $A_{1} A_{2} A_{3}=B_{1} B_{2} B_{3}$, то из равенств (2), (3) с использованием леммы 1 получаем, что

$$
A_{1}=B_{1}, \quad A_{2}=B_{2}, \quad A_{3}=B_{3}, \quad b_{1}=b_{2}, \quad c_{1}=c_{2} .
$$

Отсюда следует равенство $Q_{1}=Q_{2}$, противоречащее инъективности отображения $\varphi$. Теперь из равенств (2), (3) замечаем, что слова $A_{1} A_{2} A_{3}, B_{1} B_{2} B_{3}$ имеют общее начало длины

$$
l \geqslant \min \left\{d\left(A_{1} A_{2}\right), d\left(B_{1} B_{2}\right)\right\}
$$

и общий конец длины

$$
k \geqslant \min \left\{d\left(A_{2} A_{3}\right), d\left(B_{2} B_{3}\right)\right\} .
$$


Значит, неравенство $A_{1} A_{2} A_{3} \neq B_{1} B_{2} B_{3}$ возможно лишь в тех случаях, когда

$$
d\left(A_{1} A_{2}\right)<d\left(B_{1}\right) \text { или } d\left(B_{1} B_{2}\right)<d\left(A_{1}\right)
$$

ибо в противном случае $l+k \geqslant d\left(A_{1} A_{2} A_{3}\right)$ и потому $A_{1} A_{2} A_{3}=B_{1} B_{2} B_{3}$. В силу симметрии достаточно рассмотреть одно из неравенств условия (6).

Пусть $d\left(A_{1} A_{2}\right)<d\left(B_{1}\right)$, и тогда $d\left(A_{3}\right)>d\left(B_{2} B_{3}\right)$.

Учитывая эти соотношения, мы из равенств $(2),(3)$ получим соотношения

$$
\begin{aligned}
B_{1} & =A_{1} A_{2} B_{1}^{\prime}, & A_{3} & =A_{3}^{\prime} B_{2} B_{3}, \\
B_{1}^{\prime} & \neq \Lambda, & A_{3}^{\prime} & \neq \Lambda, \\
c_{1} A_{3}^{\prime} B_{2} & =B_{1}^{\prime} B_{2} c_{2}, & b_{1} A_{2} A_{3}^{\prime} & =A_{2} B_{1}^{\prime} b_{2} .
\end{aligned}
$$

Приравнивая в последних равенствах соответствующие буквы и учитывая утверждение (а) леммы 1 , получим, что

$$
\begin{array}{rlrl}
B_{2}=c_{2}^{s}, & A_{3}^{\prime}=A_{3}^{\prime \prime} c_{2}, & c_{1} A_{3}^{\prime \prime}=B_{1}^{\prime}, & s \geqslant 0 ; \\
A_{2}=b_{1}^{t}, & B_{1}^{\prime}=b_{1} B_{1}^{\prime \prime}, \quad A_{3}^{\prime}=B_{1}^{\prime \prime} b_{2}, \quad t \geqslant 0 .
\end{array}
$$

Отсюда видно, что $c_{1}=b_{1}, c_{2}=b_{2}, A_{3}^{\prime \prime}=B_{1}^{\prime \prime}$. Подставляя полученные данные в равенства $(2),(3)$, получим равенство. $P_{1} c P_{2}=P_{1}^{\prime} d P_{2}^{\prime}$, противоречащее инъективности $\varphi$. Таким образом, вариант I невозможен.

Рассмотрим вариант II. Здесь в зависимости от соотношений между числами $k_{i}=d\left(A_{i}\right), l_{i}=d\left(B_{i}\right), i=1,2,3$, логически возможны 6 подслучаев:

(1) $k_{1}+k_{2} \leqslant l_{1}$

(2) $k_{1} \leqslant l_{1}, l_{1}<k_{1}+k_{2}<l_{1}+l_{2}$,

(3) $k_{1} \leqslant l_{1}, k_{1}+k_{2} \geqslant l_{1}+l_{2}$.

Остальные 3 случая симметричны указанным, они получаются из 1-3 заменой $k_{i}$ на $l_{i}$ и $l_{i}$ на $k_{i}, i=1,2$. В связи с этим достаточно рассмотреть указанные 3 случая.

В первом случае $d\left(A_{1} A_{2}\right) \leqslant d\left(B_{1}\right)$, и значит, $d\left(A_{3}\right) \geqslant d\left(B_{2} B_{3}\right)$.

Отсюда и из (4), (5) получаем, что

$$
\begin{aligned}
B_{1} & =A_{1} A_{2} B_{1}^{\prime}, \quad A_{3}=A_{3}^{\prime} B_{2} B_{3}, \\
c_{1} A_{3}^{\prime} & =B_{1}^{\prime} b_{2}, \quad b_{1} A_{2} A_{3}^{\prime} B_{2}=A_{2} B_{1}^{\prime} B_{2} c_{2} .
\end{aligned}
$$

Если $A_{3}^{\prime}=\Lambda$, то $B_{1}^{\prime}=\Lambda, c_{1}=b_{2}, b_{1}=c_{2}, A_{2}=b_{1}^{s}, B_{2}=b_{1}^{t}, s, t \in \mathbf{N}_{0}$. Используя полученные данные, найдем, что

$$
\begin{array}{ll}
P_{1} c P_{2}=A_{1} b_{1}^{s} b_{2} b_{1}^{t} B_{3}, & Q_{1}=A_{1} b_{1}^{s} b_{1} b_{2} b_{1}^{t} B_{3}, \\
P_{1}^{\prime} d P_{2}^{\prime}=A_{1} b_{1}^{s} b_{1} b_{1}^{t} B_{3}, & Q_{2}=A_{1} b_{1}^{s} b_{2} b_{1} b_{1}^{t} B_{3} .
\end{array}
$$

Отсюда видно, что $P_{1}=P_{1}^{\prime}, P_{2}=P_{2}^{\prime}, c=b_{2}, d=b_{1}, b_{2} \neq b_{1}$, и, следовательно, выполняется условие $2^{\circ}$.

Если же $A_{3}^{\prime} \neq \Lambda$, то из (7) находим, что

$$
A_{3}^{\prime}=A_{3}^{\prime \prime} b_{2}, \quad B_{1}^{\prime}=c_{1} B_{1}^{\prime \prime}, \quad A_{3}^{\prime \prime}=B_{1}^{\prime \prime}, \quad b_{1}=c_{1}, \quad b_{2}=c_{2}, \quad A_{2}=b_{1}^{s}, \quad B_{2}=b_{2}^{t},
$$


где $s \geqslant 0, t \geqslant 0$. Используя полученные соотношения, мы из (4), (5) получим, что

$$
P_{1} c P_{2}=P_{1}^{\prime} d P_{2}^{\prime}=A_{1} b_{1}^{s+1} A_{3}^{\prime \prime} b_{2}^{t+1} B_{3} .
$$

Это противоречит инъективности $\varphi$.

Во втором случае

$$
d\left(A_{1}\right) \leqslant d\left(B_{1}\right), \quad d\left(B_{1}\right)<d\left(A_{1} A_{2}\right)<d\left(B_{1} B_{2}\right) .
$$

Из этих неравенств и соотношений (4), (5) получаем, что

$$
\begin{gathered}
B_{1}=A_{1} B_{1}^{\prime}, \quad A_{2}=B_{1}^{\prime} A_{2}^{\prime}, \quad A_{3}=A_{3}^{\prime} B_{3}, \quad A_{1} A_{2}=A_{1} B_{1}^{\prime} A_{2}^{\prime}, \\
B_{2}=B_{2}^{\prime} A_{3}^{\prime}, \quad A_{2}^{\prime} c_{1}=b_{2} B_{2}^{\prime}, \quad b_{1} B_{1}^{\prime} A_{2}^{\prime} A_{3}^{\prime}=B_{1}^{\prime} B_{2}^{\prime} A_{3}^{\prime} c_{2},
\end{gathered}
$$

где $A_{2}^{\prime} \neq \Lambda$. Из двух последних равенств находим, что

$$
\begin{gathered}
A_{2}^{\prime}=b_{2} A_{2}^{\prime \prime}, \quad B_{2}^{\prime}=B_{2}^{\prime \prime} c_{1}, \quad A_{2}^{\prime \prime}=B_{2}^{\prime \prime}, \quad B_{1}^{\prime}=b_{1}^{s}, \quad A_{3}^{\prime}=c_{2}^{t}, \quad s, t \geqslant 0 \\
b_{1} b_{2} A_{2}^{\prime \prime}=A_{2}^{\prime \prime} c_{1} c_{2} .
\end{gathered}
$$

Последнее равенство по лемме 2 возможно в двух случаях:

(a) $A_{2}^{\prime \prime}=\left(b_{1} b_{2}\right)^{r}, c_{1}=b_{1}, c_{2}=b_{2}$;

(b) $A_{2}^{\prime \prime}=\left(b_{1} b_{2}\right)^{r} b_{1}, c_{1}=b_{2}, c_{2}=b_{1}, r \geqslant 0$.

В случае (a)

$$
\begin{gathered}
A_{2}=b_{1}^{s} b_{2}\left(b_{1} b_{2}\right)^{r}, \quad A_{3}=b_{2}^{t} B_{3}, \quad B_{1}=A_{1} b_{1}^{s}, \quad B_{2}=\left(b_{1} b_{2}\right)^{r} b_{1} b_{2}^{t}, \\
P_{1} c P_{2}=\left(A_{1} b_{1}^{s}\right)\left(b_{2} b_{1}\right)^{r+1}\left(b_{2}^{t} B_{3}\right), \quad P_{1}^{\prime} d P_{2}^{\prime}=\left(A_{1} b_{1}^{s}\right)\left(b_{1} b_{2}\right)^{r+1}\left(b_{2}^{t} B_{3}\right), \\
Q_{1}=\left(A_{1} b_{1}^{s}\right)\left(b_{1} b_{2}\right)^{r+1} b_{1}\left(b_{2}^{t+1} B_{3}\right), \quad Q_{2}=\left(A_{1} b_{1}^{s}\right)\left(b_{2} b_{1}\right)^{r+1} b_{2}\left(b_{2}^{t+1} B_{3}\right), \quad b_{1} \neq b_{2} .
\end{gathered}
$$

Отсюда видно, что выполняется условие $5^{\circ}$.

В случае (b) получим условие $4^{\circ}$ :

$$
\begin{aligned}
& P_{1} c P_{2}=A_{1} b_{1}^{s} b_{2}\left(b_{1} b_{2}\right)^{r+1} b_{1}^{t} B_{3}, \quad P_{1}^{\prime} d P_{2}^{\prime}=A_{1} b_{1}^{s} b_{1}\left(b_{2} b_{1}\right)^{r+1} b_{1}^{t} B_{3}, \\
& Q_{1}=A_{1} b_{1}^{s}\left(b_{1} b_{2}\right)^{r+2} b_{1}^{t} B_{3}, \quad Q_{2}=A_{1} b_{1}^{s}\left(b_{2} b_{1}\right)^{r+2} b_{1}^{t} B_{3}, \quad s, t, r \geqslant 0 .
\end{aligned}
$$

В третьем случае

$$
d\left(A_{1}\right) \leqslant d\left(B_{1}\right), \quad d\left(A_{1} A_{2}\right)>d\left(B_{1} B_{2}\right),
$$

и тогда $d\left(B_{3}\right) \geqslant d\left(A_{3}\right)$. Отсюда и из $(4),(5)$ находим, что

$$
\begin{gathered}
B_{1}=A_{1} B_{1}^{\prime}, \quad A_{2}=B_{1}^{\prime} A_{2}^{\prime}, \quad B_{3}=B_{3}^{\prime} A_{3}, \quad A_{2}=A_{2}^{\prime \prime} B_{3}^{\prime}, \\
A_{2}^{\prime} c_{1}=b_{2} B_{2} B_{3}^{\prime}, \quad b_{1} B_{1}^{\prime} A_{2}^{\prime}=B_{1}^{\prime} B_{2} c_{2} B_{3}^{\prime} .
\end{gathered}
$$

Если $A_{2}^{\prime}=\Lambda$, то $B_{2} B_{3}^{\prime}=\Lambda, c_{1}=b_{2}, c_{2}=b_{1}, B_{1}^{\prime}=b_{1}^{s}, s \geqslant 0$, и потому

$$
\begin{aligned}
P_{1} c P_{2} & =A_{1} b_{1}^{s} b_{2} A_{3}, & P_{1}^{\prime} d P_{2}^{\prime} & =A_{1} b_{1}^{s+1} A_{3}, \\
Q_{1} & =A_{1} b_{1}^{k} b_{1} b_{2} A_{3}, & Q_{2} & =A_{1} b_{1}^{k} b_{2} b_{1} A_{3} .
\end{aligned}
$$


Следовательно, выполняется условие $2^{\circ}$.

Пусть $A_{2}^{\prime} \neq \Lambda$. Тогда $B_{2} B_{3}^{\prime} \neq \Lambda, A_{2}^{\prime}=b_{2} A_{2}^{\prime \prime}, B_{2} B_{3}^{\prime}=B_{2} B_{3}^{\prime \prime} c_{1}$ при $B_{3}^{\prime} \neq \Lambda$ и $B_{2}=B_{2}^{\prime} c_{1}$ при $B_{3}^{\prime}=\Lambda$.

Если $B_{3}^{\prime} \neq \Lambda$, то $B_{3}^{\prime}=B_{3}^{\prime \prime} c_{1}$ и из (8) следует, что

$$
\begin{gathered}
b_{2} A_{2}^{\prime \prime} c_{1}=b_{2} B_{2} B_{3}^{\prime \prime} c_{1}, \quad A_{2}^{\prime \prime}=B_{2} B_{3}^{\prime \prime} \\
b_{1} B_{1}^{\prime} b_{2} B_{2} B_{3}^{\prime \prime}=B_{1}^{\prime} B_{2} c_{2} B_{3}^{\prime \prime} c_{1} .
\end{gathered}
$$

Из последнего равенства видно, что

$$
B_{1}^{\prime}=b_{1}^{s}, \quad B_{3}^{\prime \prime}=c_{1}^{t}, \quad b_{1} b_{2} B_{2}=B_{2} c_{2} c_{1}, \quad s \geqslant 0, \quad t \geqslant 0
$$

Здесь в силу утверждения (b) леммы 2 снова возможны два подслучая:

(a) $B_{2}=\left(b_{1} b_{2}\right)^{r}, c_{2}=b_{1}, c_{1}=b_{2}$,

(b) $B_{2}=\left(b_{1} b_{2}\right)^{r} b_{1}, c_{2}=b_{2}, c_{1}=b_{1}, r \geqslant 0$.

В случае (a) находим, что

$$
\begin{aligned}
P_{1} c P_{2} & =\left(A_{1} b_{1}^{s}\right)\left(b_{2} b_{1}\right)^{r} b_{2}\left(b_{2}^{t+1} A_{3}\right), & P_{1}^{\prime} d P_{2}^{\prime} & =\left(A_{1} b_{1}^{s}\right) b_{1}\left(b_{2} b_{1}\right)^{r}\left(b_{2}^{t+1} A_{3}\right), \\
Q_{1} & =\left(A_{1} b_{1}^{s}\right)\left(b_{1} b_{2}\right)^{r+1}\left(b_{2}^{t+1} A_{3}\right), & Q_{2} & =\left(A_{1} b_{1}^{s}\right)\left(b_{2} b_{1}\right)^{r+1}\left(b_{2}^{t+1} A_{3}\right) .
\end{aligned}
$$

Отсюда видно, что выполняется условие $2^{\circ}$ при $r=0$ и условие $4^{\circ}$ при $r>0$. В случае (b) получим условие $5^{\circ}$, поскольку здесь

$$
\begin{aligned}
P_{1} c P_{2} & =\left(A_{1} b_{1}^{s}\right)\left(b_{2} b_{1}\right)^{r+1}\left(b_{1}^{t+1} A_{3}\right), & P_{1}^{\prime} d P_{2}^{\prime} & =\left(A_{1} b_{1}^{s}\right)\left(b_{1} b_{2}\right)^{r+1}\left(b_{1}^{t+1} A_{3}\right), \\
Q_{1} & =\left(A_{1} b_{1}^{s}\right)\left(b_{1} b_{2}\right)^{r+1} b_{1}\left(b_{1}^{t+1} A_{3}\right), & Q_{2} & =A_{1} b_{1}^{s} b_{2}\left(b_{1} b_{2}\right)^{r+1}\left(b_{1}^{t+1} A_{3}\right) .
\end{aligned}
$$

Если, наконец, $B_{3}^{\prime}=\Lambda$, то $B_{2}=B_{2}^{\prime} c_{1}$, и тогда

$$
\begin{gathered}
b_{2} A_{2}^{\prime \prime} c_{1}=b_{2} B_{2}^{\prime} c_{1}, \quad A_{2}^{\prime \prime}=B_{2}^{\prime}, \\
b_{1} B_{1}^{\prime} b_{2} A_{2}^{\prime \prime}=B_{1}^{\prime} A_{2}^{\prime \prime} c_{1} c_{2} .
\end{gathered}
$$

Отсюда находим, что

$$
B_{1}^{\prime}=b_{1}^{s}, \quad b_{1} b_{2} A_{2}^{\prime \prime}=A_{2}^{\prime \prime} c_{1} c_{2} .
$$

И снова возможны два подслучая:

(a) $A_{2}^{\prime \prime}=\left(b_{1} b_{2}\right)^{t}, c_{1}=b_{1}, c_{2}=b_{2}$,

(b) $A^{\prime \prime}=\left(b_{1} b_{2}\right)^{t} b_{1}, c_{2}=b_{1}, c_{1}=b_{2}$.

Находя, как и выше, слова $P_{1} c P_{1}, P_{1}^{\prime} d P_{2}^{\prime}, Q_{1}, Q_{2}$, мы получим, что в случае (а) выполняется условие $5^{\circ}$, в случае (b) условие $3^{\circ}$.

Лемма доказана.

Лемма 4. Если $|\Omega| \geqslant 3, u \varphi \in G(\Omega, \varepsilon)$, то для всех пар различных элементов из $\Omega$ выполняется лишь одно из условий $1^{\circ}, 2^{\circ}$. 
Доказательство. Допустим, что $a, b \in \Omega, a \neq b$ и для элементов $a, b$ выполняется условие $3^{\circ}$. Тогда

$$
\varphi(a)=A b_{1}\left(b_{2} b_{1}\right)^{r} B, \quad \varphi(b)=A b_{2}\left(b_{1} b_{2}\right)^{r} B, \quad r>0 .
$$

Выберем в $\Omega$ элемент $c \neq a, b$ и допустим, что для элементов $a, c$ выполняется одно из условий $1^{\circ}-4^{\circ}$. Тогда имеют место равенства

$$
\varphi(a)=A_{1} c_{1}\left(c_{2} c_{1}\right)^{l} B_{1}, \quad \varphi(c)=A_{1} c_{2}\left(c_{1} c_{2}\right)^{l} B_{1}, \quad c_{1} \neq c_{2}, \quad l \geqslant 0 .
$$

Заметим, что при $l=0$ выполняется условие $1^{\circ}$ или $2^{\circ}$, при $l>0$ условие $3^{\circ}$ или $4^{\circ}$.

Так как $\varphi \in G(\Omega, \varepsilon)$ и $a \varepsilon \Lambda, b \varepsilon \Lambda, c \varepsilon \Lambda$, то

$$
\varphi(a) \varepsilon \varphi(\Lambda), \quad \varphi(b) \varepsilon \varphi(\Lambda), \quad \varphi(c) \varepsilon \varphi(\Lambda) .
$$

Отсюда, по лемме 2 имеются, с точностью до симметрии, две возможности для $\varphi(\Lambda)$ :

$$
\begin{aligned}
& \varphi(\Lambda)=A\left(b_{1} b_{2}\right)^{r} B=A_{1}\left(c_{1} c_{2}\right)^{l} B_{1}, \\
& \varphi(\Lambda)=A\left(b_{2} b_{1}\right)^{r} B=A_{1}\left(c_{1} c_{2}\right)^{l} B_{1} .
\end{aligned}
$$

При этом в обоих случаях выполняются равенства

$$
\varphi(a)=A b_{1}\left(b_{2} b_{1}\right)^{r} B=A_{1} c_{1}\left(c_{2} c_{1}\right)^{l} B_{1} .
$$

Рассмотрим (11) и (12) отдельно.

В первом случае, сравнивая слова из равенств (11), (13), получаем, что если $l>0$, To

$$
b_{2}=c_{2}, \quad b_{1}=c_{1}, \quad B_{1}=B, \quad A\left(b_{1} b_{2}\right)^{r}=A_{1}\left(b_{1} b_{2}\right)^{l} .
$$

Из этих равенств и условий $3^{\circ}, 4^{\circ}$ приходим соответственно к равенствам $\varphi(b a)=$ $\varphi(c a)$ или $\varphi(b a)=\varphi(c a)$, противоречащим инъективности $\varphi$. Если же $l=0$, то $A_{1}$ имеет вид $A_{1}=A_{1}^{\prime} g c_{1}^{k}$, где $k \geqslant 0, g \neq c_{1}$. Теперь равенства (11), (13) примут вид

$$
\begin{aligned}
A\left(b_{1} b_{2}\right)^{r} B & =A_{1}^{\prime} g c_{1}^{k} B_{1}, \\
A\left(b_{1} b_{2}\right)^{r} b_{1} B & =A_{1}^{\prime} g c_{1} c_{1}^{k} B_{1} .
\end{aligned}
$$

Из них видно, что

$$
b_{2}=g, \quad b_{1}=c_{1}, \quad B=c_{1}^{k} B_{1}, \quad A\left(b_{1} b_{2}\right)^{r}=A_{1}^{\prime} g
$$

и поэтому

$$
\begin{aligned}
& \varphi(b)=A b_{2}\left(b_{1} b_{2}\right)^{r} b_{1}^{k} B_{1}, \\
& \varphi(c)=A\left(b_{1} b_{2}\right)^{r} b_{1}^{k} c_{2} B_{1} .
\end{aligned}
$$

По лемме 3 элементы $b, c$ обязаны удовлетворять одному из условий $1^{\circ}-6^{\circ}$. Так как $r>0$, условия $1^{\circ}, 2^{\circ}$ невозможны. Тогда необходимо, чтобы

$$
k=1, \quad c_{2}=b_{2}, \quad \varphi(b)=A\left(b_{2} b_{1}\right)^{r+1} B_{1}, \quad \varphi(c)=A\left(b_{1} b_{2}\right)^{r+1} B_{1},
$$


и, значит, элементы $b, c$ удовлетворяют условию $5^{\circ}$ или $6^{\circ}$. Следовательно,

$$
\{\varphi(b c), \varphi(c b)\}=\left\{A b_{2}\left(b_{1} b_{2}\right)^{r+1} B_{1}, A\left(b_{1} b_{2}\right)^{r+1} b_{1} B_{1}\right\}
$$

С другой стороны, при найденных условиях имеют место равенства

$$
\varphi(b a)=A\left(b_{1} b_{2}\right)^{r+1} B=A\left(b_{1} b_{2}\right)^{r+1} b_{1} B_{1}
$$

и мы снова пришли к противоречию с инъективностью $\varphi$.

Во втором случае, сравнивая слова из равенств (12), (13), находим, что

$$
B_{1}=c_{1}^{k} g B_{1}^{\prime}, \quad g \neq c_{1}, \quad k \geqslant 0, \quad A=A_{1}\left(c_{1} c_{2}\right)^{l} c_{1}^{k}, \quad\left(b_{1} b_{2}\right)^{r} B=g B_{1}^{\prime} .
$$

В этих условиях равенство (12) принимает вид

$$
A_{1}\left(c_{1} c_{2}\right)^{l} c_{1}^{k}\left(b_{2} b_{1}\right)^{r} B=A_{1}\left(c_{1} c_{2}\right)^{l} c_{1}^{k}\left(b_{1} b_{2}\right)^{r} B
$$

Из него следует противоречащее условию равенство $b_{1}=b_{2}$.

Таким образом, для элементов $a, c$ не может выполняться ни одно из условий $1^{\circ}-4^{\circ}$. Остается исключить условия $5^{\circ}, 6^{\circ}$. Допустим, что выполняется одно из них. Тогда

$$
\begin{aligned}
& \varphi(a)=A_{1}\left(c_{2} c_{1}\right)^{l+1} B_{1}, \\
& \varphi(c)=A_{1}\left(c_{1} c_{2}\right)^{l+1} B_{1}, \quad l \geqslant 0 .
\end{aligned}
$$

По аналогии с предыдущим случаем здесь также достаточно рассмотреть подслучаи

$$
\begin{aligned}
& \varphi(\Lambda)=A\left(b_{1} b_{2}\right)^{r} B=A_{1}\left(c_{1} c_{2}\right)^{l} c_{1} B_{1} \\
& \varphi(\Lambda)=A\left(b_{2} b_{1}\right)^{r} B=A_{1}\left(c_{1} c_{2}\right)^{l} c_{1} B_{1} .
\end{aligned}
$$

При этом в обоих случаях выполняются равенства

$$
\varphi(a)=A\left(b_{1} b_{2}\right)^{r} b_{1} B=A_{1}\left(c_{2} c_{1}\right)^{l+1} B_{1} .
$$

В первом случае, сравнивая слова из (14), (16), замечаем, что $A_{1}=A_{1}^{\prime} g c_{2}^{k}$, где $g \neq c_{2}, k \geqslant 0$, причем

$$
B=c_{2}^{k} c_{1}\left(c_{2} c_{1}\right)^{l} B_{1}, \quad A\left(b_{1} b_{2}\right)^{r}=A_{1}^{\prime} g, \quad g=b_{2}, \quad c_{2}=b_{1} .
$$

В этих условиях

$$
\begin{aligned}
& \varphi(b)=A b_{2}\left(b_{1} b_{2}\right)^{r} b_{1}^{k} c_{1}\left(b_{1} c_{1}\right)^{l} B_{1}, \\
& \varphi(c)=A\left(b_{1} b_{2}\right)^{r} b_{1}^{k} c_{1} b_{1}\left(c_{1} b_{1}\right)^{l} B_{1} .
\end{aligned}
$$

Так как в силу леммы 3 слова $b, c$ должны удовлетворять одному из условий $1^{\circ}-6^{\circ}$, имеюм две возможности: $k=0, b_{2}=b_{1}$ или $k=1, b_{2}=c_{1}$. Первая из них противоречит условию $b_{1} \neq b_{2}$, вторая приводит к равенству $\varphi(c b)=\varphi(b a)$ или к равенству $\varphi(b c)=\varphi(b a)$, противоречащим инъективности $\varphi$.

Во втором случае из сравнения слов в равенствах $(15),(16)$ получаем, что

$$
A=A_{1}, \quad b_{2}=c_{1}, \quad b_{1}=c_{2}, \quad\left(b_{2} b_{1}\right)^{r} B=\left(b_{2} b_{1}\right)^{l} b_{2} B_{1} .
$$


Если $r>l$ и $r=l+s$, то $B=b_{1}\left(b_{2} b_{1}\right)^{s-1} B$,

$$
\begin{aligned}
& \varphi(b)=A\left(b_{2} b_{1}\right)^{l+1}\left(b_{2} b_{1}\right)^{s-1} b_{2} B, \\
& \varphi(c)=A\left(b_{2} b_{1}\right)^{l+1} b_{1}\left(b_{2} b_{1}\right)^{s-1} B .
\end{aligned}
$$

Отсюда видно, что для элементов $b, c$ выполняется условие $1^{\circ}$ или $2^{\circ}$ при $s=1$ и условие $3^{\circ}$ или $4^{\circ}$ при $s>1$. Во всех этих случаях среди элементов $\varphi(b c), \varphi(c b)$ есть элемент $A\left(b_{2} b_{1}\right)^{r+1} B$, совпадающий с $\varphi(b a)$, и мы пришли к противоречию с условием инъективности $\varphi$. K такому же результату ведет и случай $r \leqslant l$.

Таким образом, доказано, что для элементов $a, b$ не может выполняться условие $3^{\circ}$. Из симметричных соображений не может для них выполняться и условие $4^{\circ}$.

Допустим теперь, что для $a, b$ выполняется условие $5^{\circ}$. Тогда, по доказанному, для $a, c$ не могут выполняться условия $3^{\circ}, 4^{\circ}$. Допустим, что для них выполняется условие $1^{\circ}$ или $2^{\circ}$. Тогда

$$
\varphi(a)=A_{1} c_{1} B_{1}, \quad \varphi(c)=A_{1} c_{2} B_{1}, \quad c_{1} \neq c_{2}
$$

Отсюда и из условия $5^{\circ}$ для $a, b$ из леммы 2 следует, что $\varphi(\Lambda)=A_{1} B_{1}$ и $\varphi(\Lambda)=$ $A\left(b_{1} b_{2}\right)^{r} b_{1} B$ или $\varphi(\Lambda)=A b_{2}\left(b_{1} b_{2}\right)^{r} B$. Значит, с точностью до симметрии достаточно рассмотреть два случая

$$
\begin{aligned}
& \varphi(\Lambda)=A\left(b_{1} b_{2}\right)^{r} b_{1} B=A_{1} B_{1} \\
& \varphi(\Lambda)=A b_{2}\left(b_{1} b_{2}\right)^{r} B=A_{1} B_{1} .
\end{aligned}
$$

Причем в обоих случаях

$$
\varphi(a)=A\left(b_{2} b_{1}\right)^{r+1} B=A_{1} c_{1} B_{1} .
$$

В первом случае из сравнений слов в равенствах (17), (19) получаем, что

$$
\begin{gathered}
B_{1}=c_{1}^{k} g B_{1}^{\prime}, \quad k \geqslant 0, \quad g \neq c_{1}, \quad b_{1}=g, \quad b_{2}=c_{1}, \\
A=A_{1} b_{2}^{k}, \quad\left(b_{1} b_{2}\right)^{r} b_{1} B=b_{1} B_{1}^{\prime} .
\end{gathered}
$$

Тогда

$$
\begin{aligned}
& \varphi(b)=A_{1} b_{2}^{k}\left(b_{1} b_{2}\right)^{r+1} B, \\
& \varphi(c)=A_{1} c_{2} b_{2}^{k}\left(b_{1} b_{2}\right)^{r} b_{1} B .
\end{aligned}
$$

Так как для элементов $b, c$ должно выполняться одно из условий $1^{\circ}-6^{\circ}$, возможны подслучаи $k=0, c_{2}=b_{1}$ и $k=1, b_{1}=c_{2}$. При этом, соответственно,

$$
\begin{aligned}
& \varphi(b)=\left(A_{1} b_{1}\right) b_{2}\left(b_{1} b_{2}\right)^{r} B \\
& \varphi(c)=\left(A_{1} b_{1}\right)\left(b_{1} b_{2}\right)^{r} b_{1} B
\end{aligned}
$$

и

$$
\begin{aligned}
& \varphi(b)=A_{1} b_{2}\left(b_{1} b_{2}\right)^{r+1} B, \\
& \varphi(c)=A_{1}\left(b_{1} b_{2}\right)^{r+1} b_{1} B .
\end{aligned}
$$


В обоих подслучаях для элементов $b, c$ выполняется условие $3^{\circ}$ или $4^{\circ}$. Однако по доказанному выше это невозможно.

Во втором случае из сравнения слов в (18), (19) получаем, что

$$
\begin{gathered}
A_{1}=A_{1}^{\prime} g c_{1}^{k}, \quad g \neq c, \quad k \geqslant 0, \quad g=b_{2}, \quad c_{1}=b_{1}, \\
A\left(b_{2} b_{1}\right)^{r}=A_{1}^{\prime}, \quad B=b_{1}^{k} B_{1} .
\end{gathered}
$$

Далее приходим к двум возможностям: $k=0, c_{2}=b_{1}$ или $k=1, b_{1}=c_{2}$.

Для каждой из них элементы $b, c$ удовлетворяют условию $3^{\circ}$ или $4^{\circ}$, и мы снова пришли к противоречию с доказанным выше.

Теперь докажем, что для всех пар различных элементов из $\Omega$ выполняется одно и то же условие из $1^{\circ}, 2^{\circ}$. Если же это не так, то найдутся такие три элемента $a, b, c \in \Omega$, что для $a, b$ выполняется условие $1^{\circ}$, а для $a, c$ условие $2^{\circ}$. Тогда

$$
\begin{aligned}
& \varphi(a)=A b_{1} B, \quad \varphi(b)=A b_{2} B, \quad \varphi(\Lambda)=A B, \quad b_{1} \neq b_{2}, \\
& \varphi(a b)=A b_{1} b_{2} B, \quad \varphi(b a)=A b_{2} b_{1} B \\
& \varphi(a)=A_{1} c_{1} B_{1}, \quad \varphi(c)=A_{1} c_{2} B_{1}, \quad \varphi(\Lambda)=A_{1} B_{1}, \quad c_{1} \neq c_{2}, \\
& \varphi(a c)=A_{1} c_{2} c_{1} B_{1}, \quad \varphi(c a)=A_{1} c_{1} c_{2} B_{1} .
\end{aligned}
$$

Отыскивая в словах

$$
\begin{aligned}
& \varphi(\Lambda)=A B=A_{1} B_{1}, \\
& \varphi(a)=A b_{1} B=A_{1} c_{1} B_{1},
\end{aligned}
$$

первые слева различные буквы, получим, что

$$
B=b_{1}^{k} g B^{\prime}, \quad k \geqslant 0, \quad g \neq b_{1}
$$

(при этом не исключается случай $B=b_{1}^{k}, g=\Lambda, B^{\prime}=\Lambda$ ), и

$$
B_{1}=c_{1}^{l} h B_{1}^{\prime}, \quad l \geqslant 0, \quad h \neq c_{1} .
$$

Теперь из равенств (20), (21) следует, что

$$
A b_{1}^{k}=A_{1} c_{1}^{l}, \quad B_{1}^{\prime}=B^{\prime}, \quad g=h, \quad b_{1}=c_{1} .
$$

Пусть $k \geqslant l$, т. е. $k=l+s, s \geqslant 0$. Тогда

$$
A_{1}=A b_{1}^{s}, \quad B=b_{1}^{s} B_{1}, \quad \varphi(b)=A b_{2} b_{1}^{s} B_{1}, \quad \varphi(c)=A b_{1}^{s} c_{2} B_{1} .
$$

Так как элементы $b, c$ должны удовлетворять условию $1^{\circ}$ или $2^{\circ}$, из последних двух равенств, учитывая, что $c_{2} \neq b_{1}$, получаем равенство $s=0$, т. е.

$$
A=A_{1}, \quad B=B_{1}, \quad \varphi(c)=A c_{2} B .
$$

В силу симметрии к такому же выводу приводит и случай $l \geqslant k$.

Допустим теперь, что элементы $b, c$ удовлетворяют условию $1^{\circ}$, т. $\mathrm{e}$

$$
\begin{aligned}
& \varphi(b c)=A b_{2} c_{2} B, \\
& \varphi(c b)=A c_{2} b_{2} B .
\end{aligned}
$$


Рассмотрим слово $Q=a b c \in \Omega^{*}$. Так как $\varepsilon(a b c)=\{a b, a c, b c\}$, справедливо включение

$$
\varepsilon(\varphi(Q)) \supseteq\left\{A b_{1} b_{2} B, A c_{2} b_{1} B, A b_{2} c_{2} B\right\} .
$$

Однако нетрудно видеть, что такого слова $\varphi(Q)$ не существует (поскольку $b_{1}, b_{2}, c_{2}$ попарно различны).

Пусть элементы $b, c$ удовлетворяют условию $2^{\circ}$. Здесь, выбрав $Q=a c b$, мы придем к противоречию точно так же, как и в случае выполнения условия $1^{\circ}$. Лемма доказана.

Теперь докажем теоремы 1,2 .

Доказательство теоремы 1. Очевидно, что отображения вида (3) и (4) удовлетворяют условию (2) и поэтому принадлежат множеству $G\left(\Omega, \Omega_{1}, \varepsilon\right)$. Доказывать будем обратное утверждение.

Пусть $|\Omega| \geqslant 3$ и $\varphi \in G\left(\Omega, \Omega_{1}, \varepsilon\right)$.

Согласно лемме 4 любое отображение $\varphi \in G\left(\Omega, \Omega_{1}, \varepsilon\right)$ имеет вид (3) или (4) на всех словах длины $l \leqslant 1$ и словах вида $a_{1} a_{2}$ при $a_{1} \neq a_{2}$.

Выясним, какие значения могут иметь слова $\varphi(a a), a \in \Omega$. Выберем в $\Omega$ три различных элемента $a, b, c$. По лемме 4

$$
\begin{gathered}
\varphi(a)=A b_{1} B, \quad \varphi(b)=A b_{2} B, \quad \varphi(c)=A b_{3} B \\
\{\varphi(a b), \varphi(b a)\}=\left\{A b_{1} b_{2} B, A b_{2} b_{1} B\right\}, \quad\{\varphi(a c), \varphi(c a)\}=\left\{A b_{1} b_{3} B, A b_{3} b_{1} B\right\}
\end{gathered}
$$

при некоторых различных $b_{1}, b_{2}, b_{3}$.

Из двух последних равенств в силу леммы 2 следует, что $\varphi(a b a)$ равно $A b_{1} b_{2} b_{1} B$ или $A b_{2} b_{1} b_{2} B, \varphi(a c a)$ равно $A b_{1} b_{3} b_{1} B$ или $A b_{3} b_{1} b_{3} B$. Отсюда и из условий

$$
\varphi(a b a) \varepsilon \varphi(a a), \quad \varphi(a c a) \varepsilon \varphi(a a)
$$

находим единственную возможность:

$$
\varphi(a b a)=A b_{1} b_{2} b_{1} B, \quad \varphi(a c a)=A b_{1} b_{3} b_{1} B, \quad \varphi(a a)=A b_{1} b_{1} B .
$$

В итоге доказано, что утверждение теоремы 1 справедливо для всех слов $P$ длины $l \leqslant 2$, а также для слов вида $a b a, a \neq b$.

Теперь применим индукцию по длине слов. Допустим, что

$$
\varphi(P)=A P^{\sigma} B
$$

для всех слов длины $k \geqslant 2$ и рассмотрим слова длины $k+1$.

Сначала будем искать слова $\varphi(P)$ для слов $P$ из $\Omega^{k+1}$, отличных от слов вида $a^{k+1}$ и $a^{s} b^{t}, s+t=k+1$. Любое такое слово можно представить в виде

$$
P=a^{r} b^{l} c^{s} P_{1}, \quad r, l, s>0, \quad a \neq b, \quad c \neq b .
$$

Для слов

$$
Q_{1}=a^{r-1} b^{l} c^{s} P_{1}, \quad Q_{2}=a^{r} b^{l-1} c^{s} P_{1}, \quad Q_{3}=a^{r} b^{l} c^{s-1} P_{1}
$$

из $\varepsilon(P)$ по предположению индукции

$$
\varphi\left(Q_{i}\right)=A Q_{i}^{\sigma} B, \quad i=1,2,3 .
$$


Обозначив $a^{\sigma}=b_{1}, b^{\sigma}=b_{2}, c^{\sigma}=b_{3}$, получим, что

$$
\begin{aligned}
& \varphi\left(Q_{1}\right)=A b_{1}^{r-1} b_{2}^{l} b_{3}^{s} P_{1}^{\sigma} B, \\
& \varphi\left(Q_{2}\right)=A b_{1}^{r} b_{2}^{l-1} b_{3}^{s} P_{1}^{\sigma} B, \\
& \varphi\left(Q_{3}\right)=A b_{1}^{r} b_{2}^{l} b_{3}^{s-1} P_{1}^{\sigma} B .
\end{aligned}
$$

Так как $\varphi(P) \varepsilon \varphi\left(Q_{1}\right)$ и $\varphi(P) \varepsilon \varphi\left(Q_{2}\right)$, по лемме 3 для $\varphi(P)$ имеются лишь две возможности:

$$
\varphi(P)=A b_{1}^{r} b_{2}^{l} b_{3}^{s} P_{1}^{\sigma} B
$$

или

$$
\varphi(P)=A b_{1}^{r-1} b_{2} b_{1} b_{2}^{l-1} b_{3}^{s} P_{1}^{\sigma} B .
$$

В первой из них мы получаем равенство (22), во второй противоречие с условием $\varphi(P) \varepsilon \varphi\left(Q_{3}\right)$. Итак, равенство (22) справедливо для всех слов вида (23).

Пусть теперь $P=a^{r} b^{l}, r \geqslant 1, l \geqslant 1, d(P) \geqslant 3$.

Из тех же соображений и в тех же обозначениях, что и выше, при $Q_{1}=a^{r-1} b^{l}$, $Q_{2}=a^{r} b^{l-1}$, получим, что

$$
\varphi(P)=A P^{\sigma} B
$$

или

$$
\varphi(P)=A b_{1}^{r-1} b_{2} b_{1} b_{2}^{l-1} B .
$$

Так как $d(P) \geqslant 3$, то $r-1>0$ или $l-1>0$. Тогда по доказанному в предыдущем случае

$$
A b_{1}^{r-1} b_{2} b_{1} b_{2}^{l-1} B=\varphi\left(a^{r-1} b a b^{l-1}\right) .
$$

А так как $\varphi$ инъективно, остается лишь первая возможность, т.е. выполнено равенство (22).

Пусть теперь $P=a^{k+1}, k+1 \geqslant 3$. В этом случае воспользуемся двумя вспомогательными словами длины $k+2$ :

$$
P_{1}=a^{k} b a, \quad P_{2}=a b a^{k} .
$$

Так как $\varepsilon\left(P_{1}\right) \supseteq\left\{a^{k} b, a^{k-1} \dot{b} a\right\}$, справедливы включения

$$
\varepsilon\left(\varphi\left(P_{1}\right)\right) \supseteq\left\{\varphi\left(a^{k} b\right), \varphi\left(a^{k-1} b a\right)\right\} \supseteq\left\{A b_{1}^{k} b_{2} B, A b_{1}^{k-1} b_{2} b_{1} B\right\} .
$$

Отсюда по лемме 3 получаем, что

$$
\varphi\left(P_{1}\right)=A b_{1}^{k-1} b_{2} b_{1} b_{2} B
$$

или

$$
\varphi\left(P_{1}\right)=A b_{1}^{k-1} b_{1} b_{2} b_{1} B
$$

Аналогично находим, что

$$
\varphi\left(P_{2}\right)=A b_{1} b_{2} b_{1} b_{1}^{k-1} B
$$

или

$$
\varphi\left(P_{2}\right)=A b_{2} b_{1} b_{2} b_{1}^{k-1} B
$$


Рассматривая полученные четыре возможности и учитывая, что $a^{k+1} \varepsilon P_{1}, a^{k+1} \varepsilon P_{2}$, мы для $\varphi\left(a^{k+1}\right)$ формально найдем три варианта: $\varphi\left(a^{k+1}\right)=A b_{1}^{k+1} B$ при любом $k \geqslant 2$ и $\varphi\left(a^{k+1}\right)=A b_{1} b_{2} b_{1} B$ или $A b_{2} b_{1} b_{2} B$ при $k=2$.

Два последних варианта отпадают в силу инъективности $\varphi$, поскольку уже доказано, что

$$
A b_{1} b_{2} b_{1} B=\varphi(a b a), \quad A b_{2} b_{1} b_{2} B=\varphi(b a b) .
$$

Итак, равенство (22) верно и в этом случае.

Нетрудно видеть, что та же схема доказательства проходит и при замене формулы (22) на формулу

$$
\varphi(P)=A \mu\left(P^{\sigma}\right) B
$$

Теорема доказана.

Доказательство теоремы 2. Нетрудно проверить, что указанные в ней отображения удовлетворяют условию (2) и поэтому содержатся в $G\left(\Omega, \Omega_{1}, \varepsilon\right)$.

Докажем, что в $G\left(\Omega, \Omega_{1}, \varepsilon\right)$ других отображений нет.

Напомним, что здесь $|\Omega|=2$. Пусть $\Omega=\{0,1\}$.

Рассмотрим ряд случаев.

I. Элементы 0,1 удовлетворяют условию $1^{\circ}$ или $2^{\circ}$. Пусть

$$
\begin{aligned}
& \varphi(\Lambda)=A B, \\
& \varphi(0)=A b_{1} B, \\
& \varphi(1)=A b_{2} B .
\end{aligned}
$$

В этом случае доказательство проводится по схеме доказательства теоремы 1. В нем условие $|\Omega|>2$ существенно использовалось лишь при нахождении $\varphi(a a)$ и $\varphi(b b)$, т. е. в нашем случае $\varphi(00), \varphi(11)$. Здесь эти слова находятся сложнее. Докажем, что

$$
\varphi(11)=A b_{2} b_{2} B, \quad \varphi(00) A b_{1} b_{1} B .
$$

Из условий $1^{\circ}, 2^{\circ}$ и леммы 3 получаем, что

$$
\{\varphi(010), \varphi(101)\}=\left\{A b_{1} b_{2} b_{1} B, A b_{2} b_{1} b_{2} B\right\} .
$$

Рассмотрим две из этих возможностей отдельно.

(a) $\varphi(010)=A b_{1} b_{2} b_{1} B, \varphi(101)=A b_{2} b_{1} b_{1} B$.

Будем находить $\varphi(11)$ из условия

$$
\varphi(101) \varepsilon \varphi(11) \varepsilon \varphi(1)
$$

то есть

$$
A \underline{b_{2} b_{1} b_{2}} B \varepsilon \varphi(11) \varepsilon A b_{2} B .
$$

Здесь и далее мы подчеркиваем некоторые вхождения букв $b_{1}$ и $b_{2}$.

При переходе от слова $A b_{2} b_{1} b_{2} B$ к $\varphi(11)$ не могло удалиться ни одно из подчеркнутых вхождений буквы $b_{2}$ в силу инъективности отображения $\varphi$. Если удалилось подчеркнутое вхождение $b_{1}$, то $\varphi(11)=A b_{2} b_{2} B$. Теперь допустим, что удалялась буква из подслова $A$, то есть $A=A_{1} c A_{2}$ и $\varphi(11)=A_{1} A_{2} b_{2} b_{1} b_{2} B$.

2 Дискретная математика, т.11 №2 
Из условия $\varphi(11) \varepsilon A b_{2} B$ видно, что при переходе от $\varphi(11)$ к $A b_{2} B$ может удаляться лишь буква из подслова $A_{1} A_{2} b_{2} b_{1}$. Если удалить $b_{1}$, то получаем, что

$$
A_{1} A_{2} b_{2} b_{2} B=A_{1} c A_{2} b_{2} B \text {. }
$$

Отсюда по лемме 2

$$
c=b_{2}, \quad A_{2}=b_{2}^{k}, \quad k \geqslant 0, \quad A=b_{2}^{k+1}
$$

и $\varphi(11)=A b_{1} b_{2} B$, что противоречит инъективности $\varphi$.

Пусть удалено $b_{2}$. Тогда

$$
A_{1} A_{2} b_{1} b_{2} B=A_{1} c A_{2} b_{2} B
$$

и

$$
c=b_{1}, \quad A_{2}=b_{1}^{k}, \quad k \geqslant 0, \quad A=A_{1} b_{1}^{k+1}, \quad \varphi(11)=A_{1} b_{1}^{k} b_{2} b_{1} b_{2} B .
$$

Пусть удалена еще одна буква из $A$. Тогда $A=A_{1}^{\prime} d A_{1}^{\prime \prime} c A_{2}$ или $A=A_{1} c A_{2}^{\prime} d A_{2}^{\prime \prime}$, где $d$ - вторая удаляемая из $A$ буква. В этих случаях соответственно получаем, что

(1) $\varphi(11)=A_{1}^{\prime} d A_{1}^{\prime \prime} A_{2} b_{2} b_{1} b_{2} B$ и $A_{1}^{\prime} A_{1}^{\prime \prime} A_{2} b_{2} b_{1}=A_{1}^{\prime} d A_{1}^{\prime \prime} c A_{2}$,

(2) $\varphi(11)=A_{1} A_{2}^{\prime} d A_{2}^{\prime \prime} b_{2} b_{1} b_{2} B$ и $A_{1} A_{2}^{\prime} A_{2}^{\prime \prime} b_{2} b_{1}=A_{1} c A_{2}^{\prime} d A_{2}^{\prime \prime}$.

В случае 1 находим, что $A_{1}^{\prime \prime}=d^{k}, k \geqslant 0$, и $A_{2} b_{2} b_{1}=d c A_{2}$. Отсюда, по лемме 2 ,

$$
A_{2}=\left(b_{2} b_{1}\right)^{l}, \quad l \geqslant 0, \quad b_{2}=d, \quad b_{1}=c
$$

или

$$
A_{2}=b_{1}\left(b_{2} b_{1}\right)^{l}, \quad l \geqslant 0, \quad b_{1}=d, \quad b_{2}=c .
$$

Аналогично в случае 2

$$
A_{2}^{\prime}=c^{k}, \quad k \geqslant 0, \quad A_{2}^{\prime \prime}=\left(b_{2} b_{1}\right)^{l}, \quad c=b_{2}, \quad d=b_{1}
$$

или

$$
A_{2}^{\prime \prime}=b_{1}\left(b_{2} b_{1}\right)^{l}, \quad c=b_{1}, \quad d=b_{2} .
$$

В итоге для $\varphi(11)$, кроме требуемого значения $A b_{2} b_{2} B$, имеются пока еще пять возможностей. Запишем их в более простом виде и в несколько ином порядке:

(1) $A=A^{\prime} b_{1}, \varphi(11)=A^{\prime} b_{2} b_{1} b_{2} B$,

(2) $A=A^{\prime}\left(b_{2} b_{1}\right)^{l+1}, \varphi(11)=A^{\prime} b_{2}\left(b_{2} b_{1}\right)^{l+1} b_{2} B, l \geqslant 0$,

(3) $A=A^{\prime}\left(b_{2} b_{1}\right)^{l+1}, \varphi(11)=A^{\prime} b_{1}\left(b_{2} b_{1}\right)^{l+1} b_{2} B, l \geqslant 0$,

(4) $A=A^{\prime}\left(b_{1} b_{2}\right)^{l+1} b_{1}, \varphi(11)=A^{\prime} b_{2}\left(b_{1} b_{2}\right)^{l+2} B, l \geqslant 0$,

(5) $A=A^{\prime}\left(b_{1} b_{2}\right)^{l+1} b_{1}, \varphi(11)=A^{\prime} b_{1}\left(b_{1} b_{2}\right)^{l+2} B, l \geqslant 0$. 
Кроме того, в силу симметрии имеются еще 5 возможностей для $\varphi(11)$, связанных с удалением букв из первой части слова $A b_{2} b_{1} b_{2} B$.

Докажем, что выписанные варианты 1-5 ведут к противоречию. Предварительно заметим, что варианты 4, 5 являются частными видами соответственно вариантов 1, 3. Поэтому далее достаточно рассмотреть варианты 1-3. Для приведения к противоречию мы в каждом случае будем находить последовательно слова $\varphi(110), \varphi(011), \varphi(1101), \varphi(1011), \varphi(111)$.

Проделаем это подробно, например, для варианта 2. При этом будем пока считать, что для элементов 0,1 выполняется условие $1^{\circ}$, т. е.

$$
\varphi(01)=A b_{1} b_{2} B, \quad \varphi(10)=A b_{2} b_{1} B .
$$

Так как $110 \varepsilon 10$ и $110 \varepsilon 11$, по свойству (2) для $\varphi$

$$
\varphi(110) \varepsilon A^{\prime} b_{2}\left(b_{2} b_{1}\right)^{l+1} b_{2} B_{1}, A^{\prime}\left(b_{2} b_{1}\right)^{l+1} b_{2} b_{1} B .
$$

Отсюда, по лемме 2,

$$
\varphi(110)=A^{\prime} b_{2}\left(b_{2} b_{1}\right)^{l+2} B
$$

или

$$
\varphi(110)=A^{\prime} b_{2}\left(b_{1} b_{2}\right)^{l+2} B=A b_{2} b_{1} b_{2} B .
$$

Последнее равенство противоречит инъективности $\varphi$, поскольку $A b_{2} b_{1} b_{2} B=\varphi(101)$, остается первое.

Аналогично из $011 \varepsilon 01,11$ находим, что

$$
\varphi(011)=A^{\prime} b_{2}\left(b_{2} b_{1}\right)^{l+1} b_{1} b_{2} B .
$$

Так как $1101 \varepsilon 110$ и $1101 \varepsilon 111$, то

$$
\varphi(1101) \varepsilon A^{\prime} b_{2}\left(b_{2} b_{1}\right)^{l+2} B, A^{\prime}\left(b_{2} b_{1}\right)^{l+1} b_{2} b_{1} b_{2} B .
$$

Отсюда, по лемме 2,

$$
\varphi(1101)=A^{\prime}\left(b_{2} b_{1}\right)^{l+3} b=A b_{2} b_{1} b_{2} b_{1} B \text { или } A^{\prime} b_{2}\left(b_{2} b_{1}\right)^{l+2} b_{2} B .
$$

Аналогично из соотношений $1011 \varepsilon 101$ и $1011 \varepsilon 011$ получим, что

$$
\varphi(1011)=A b_{2} b_{1} b_{1} b_{2} B \text { или } A^{\prime} b_{2}\left(b_{2} b_{1}\right)^{l+2} b_{2} B \text {. }
$$

Заметим, что в силу леммы 2

$$
\{\varphi(1010), \varphi(0101)\} \subset\left\{A b_{2} b_{1} b_{2} b_{1} B, A b_{1} b_{2} b_{1} b_{2} B\right\},
$$

поскольку $1010 \varepsilon 010,1010 \varepsilon 101,0101 \varepsilon 101$ и $0101 \varepsilon 101$.

С учетом этого факта из (24), (25) получаем, что

$$
\begin{aligned}
& \varphi(1101)=A^{\prime} b_{2}\left(b_{2} b_{1}\right)^{l+2} b_{2} B, \\
& \varphi(1011)=A b_{2} b_{1} b_{1} b_{2} B .
\end{aligned}
$$

Из последних равенств, учитывая, что $1101 \varepsilon 111$ и $1011 \varepsilon 111$, с использованием леммы 2 находим, что

$$
\varphi(111)=A b_{2} b_{1} b_{2} B
$$


или

$$
\varphi(111)=A^{\prime} b_{2}\left(b_{2} b_{1}\right)^{l+1} b_{1} b_{2} B .
$$

В первом случае $\varphi(111)=\varphi(101)$, во втором $\varphi(111)=\varphi(011)$. Получили противоречие с инъективностью $\varphi$.

Из проведенных рассуждений видно, что смена условия $1^{\circ}$ для элементов 0,1 на условие $2^{\circ}$ приведет к перестановкам слов $\varphi(011), \varphi(110), \varphi(1011), \varphi(1101)$. Противоречие же сохранится.

Таким образом, доказано, что $\varphi(11)=A b_{2} b_{2} B$.

Из соображений симметрии можно заключить, что $\varphi(00)=A b_{1} b_{1} B$.

Заметим, что в силу симметрии к противоречию приведут и не выписанные нами значения $\varphi(11)$, полученные из слова $A b_{2} b_{1} b_{2} B$ удалением букв подслова $B$.

(b) $\varphi(101)=A b_{1} b_{2} b_{1} B, \varphi(010)=A b_{2} b_{1} b_{2} B$.

Будем искать $\varphi(11)$, исходя из соотношений

$$
A \underline{b_{1} b_{2} b_{1}} B \varepsilon \varphi(11) \varepsilon \varphi(1)=A b_{2} B .
$$

Если при переходе от $A b_{1} b_{2} b_{1} B$ к $\varphi(11)$ удалялось одно из подчеркнутых вхождений буквы $b_{i}$, то при $i=1$ получится противоречие с инъективностью $\varphi$, а при $i=2$ с условием

$$
\varphi(11) \varepsilon A b_{2} B
$$

Пусть удалялась буква из подслова $A$. Тогда из соотношения (26) следует, что при переходе от $\varphi(11)$ к $A b_{2} B$ должна удаляться буква из подслова $B$. Таким образом,

$$
A=A_{1} c_{1} A_{2}, \quad B=B_{1} c_{2} B_{2}, \quad \varphi(11)=A_{1} A_{2} b_{1} b_{2} b_{1} B, \quad \varphi(1)=A_{1} A_{2} b_{1} b_{2} b_{1} B_{1} B_{2} .
$$

Отсюда следует, что

$$
A_{1} A_{2} b_{1}=A_{1} c_{1} A_{2}, \quad B_{1} c_{2} B_{2}=b_{1} B_{1} B_{2},
$$

и потому

$$
c_{1}=b_{1}, \quad A_{2}=b_{1}^{k}, \quad c_{2}=b_{1}, \quad B_{1}=b_{1}^{l}, \quad k \geqslant 0, \quad l \geqslant 0 .
$$

Следовательно,

$$
\varphi(11)=\left(A_{1} b_{1}^{k} b_{1}\right) b_{2}\left(b_{1} B\right)=A b_{2} b_{1} B,
$$

что противоречит инъективности $\varphi$.

Из симметрии следуе', что к противоречию придем и в том случае, когда при переходе от $A b_{1} b_{2} b_{1} B$ сначала удаляется буква из $B$, затем из $A$.

Этим рассмотрение случая I закончено, поскольку индукция от слов длины 2 ко всем словам для этого случая проведена при доказательстве теоремы 1.

II. Элементы 0,1 удовлетворяют условию $3^{\circ}$. В этом случае

$$
\begin{aligned}
\varphi(0) & =A b_{1}\left(b_{2} b_{1}\right)^{r} B, \quad \varphi(1)=A b_{2}\left(b_{1} b_{2}\right)^{r} B, \quad r>0, \\
\varphi(01) & =A\left(b_{1} b_{2}\right)^{r+1} B, \quad \varphi(10)=A\left(b_{2} b_{1}\right)^{r+1} B .
\end{aligned}
$$

Здесь из леммы 2 следует, что

$$
\{\varphi(010), \varphi(101)\}=\left\{A b_{1}\left(b_{2} b_{1}\right)^{r+1} B, A b_{2}\left(b_{1} b_{2}\right)^{r+1} B\right\} .
$$


В связи с этим рассмотрим два подслучая.

(a) $\varphi(010)=A b_{1}\left(b_{2} b_{1}\right)^{r+1} B, \varphi(101)=A b_{2}\left(b_{1} b_{2}\right)^{r+1} B$.

В этом случае, как и в случае I, сначала необходимо найти $\varphi(11), \varphi(00)$. Буди искать $\varphi(11)$ из условий

$$
\varphi(101)=A \underline{b_{2}\left(b_{1} b_{2}\right)^{r+1}} B \varepsilon \varphi(11) \varepsilon A b_{2}\left(b_{1} b_{2}\right)^{r} B=\varphi(1) .
$$

Если при переходе от $A b_{2}\left(b_{1} b_{2}\right)^{r+1} B$ к $\varphi(11)$ удалить первую или последнюю букву слова $b_{2}\left(b_{1} b_{2}\right)^{r+1}$, то придем к противоречию с инъективностью $\varphi$. Значит, в подслове $b_{2} \overline{\left(b_{1} b_{2}\right)^{r+1}}$ может быть удалена лишь какая-то внутренняя буква. При этом $\varphi(11)$ будет принимать одно из значений

$$
\varphi(11)=A b_{2}\left(b_{1} b_{2}\right)^{s} b_{1}\left(b_{1} b_{2}\right)^{t} \text { или } A b_{2}\left(b_{1} b_{2}\right)^{s} b_{2}\left(b_{1} b_{2}\right)^{t} B
$$

где $s+t+r>0$.

Если же удаляется буква из подслова $A$, то, как следует из условия

$$
\varphi(11) \varepsilon A b_{2}\left(b_{1} b_{2}\right)^{r} B,
$$

при переходе от $\varphi(11)$ к $A b_{2}\left(b_{1} b_{2}\right)^{r} B$ должна будет удаляться некоторая буква из слова $A b_{2} b_{1}$. Здесь мы находимся точно в таких же условиях, что и в случае I(a) с той лишь разницей, что здесь вместо слова $B$ из I(a) выступает слово $\left(b_{1} b_{2}\right)^{r} B$.

Поэтому здесь мы получим сходные возможности $1^{\prime}-5^{\prime}$. Для их описания достаточно в 1-5 заменить $B$ на $\left(b_{1} b_{2}\right)^{r} B$. Далее по той же схеме, что и в I(a), все эти варианты для $\varphi(11)$ исключаются приведением к противоречию с инъективностью $\varphi$.

Таким образом, для $\varphi(11)$ остаются лишь значения из (28). Допустим, что имеет место первое равенство из (28). Тогда из условий $110 \varepsilon 10110 \varepsilon 11$ и $011 \varepsilon 01,011 \varepsilon 11$ находим, что

$$
\varphi(110)=A\left(b_{2} b_{1}\right)^{s} b_{2} b_{1} b_{1}\left(b_{2} b_{1}\right)^{t} B, \quad \varphi(011)=A\left(b_{1} b_{2}\right)^{s} b_{1} b_{2} b_{1}\left(b_{1} b_{2}\right)^{t} B .
$$

Так как $11011 \varepsilon 011$ и $11011 \varepsilon 110$, система уравнений

$$
x \varepsilon \varphi(110), \quad x \varepsilon \varphi(011)
$$

разрешима. Однако из (29) видно, что это возможно лишь при $t=0$. Однако при $t=0$ получим равенство $\varphi(011)=\varphi(010)$, противоречащее инъективности $\varphi$.

Таким образом, $\varphi(11)$ может иметь лишь следующий вид:

$$
\varphi(11)=A b_{2}\left(b_{1} b_{2}\right)^{s} b_{2}\left(b_{1} b_{2}\right)^{t} B, \quad s+t>0 .
$$

Отсюда по симметрии $\varphi(00)$ имеет вид

$$
\varphi(00)=A b_{1}\left(b_{2} b_{1}\right)^{l} b_{1}\left(b_{2} b_{1}\right)^{m} B, \quad l+m>0 .
$$

Докажем еще, что $s=l$. Допустим, что $s>l$, то есть $s=l+k, k>0$, и тогда $m=t+k$. Составим таблицу значений слов $\varphi(P)$ для некоторых слов $P$ длины 3 (по использованной выше методике). Получим следующую таблицу:

\begin{tabular}{|c|c|}
\hline$P$ & $\varphi(P)$ \\
\hline 100 & $A\left(b_{2} b_{1}\right)^{l} b_{2} b_{1}\left(b_{1} b_{2}\right)^{k} b_{1}\left(b_{2} b_{1}\right)^{t} B$ \\
101 & $A\left(b_{2} b_{1}\right)^{l} b_{2} b_{1}\left(b_{2} b_{1}\right)^{k} b_{2}\left(b_{1} b_{2}\right)^{t} B$ \\
110 & $A\left(b_{2} b_{1}\right)^{l}\left(b_{2} b_{1}\right)^{k} b_{2} b_{2} b_{1}\left(b_{2} b_{1}\right)^{t} B$ \\
111 & $A\left(b_{2} b_{1}\right)^{l}\left(b_{2} b_{1}\right)^{k} b_{2} b_{2} b_{2}\left(b_{1} b_{2}\right)^{t} B$ \\
\hline
\end{tabular}


Теперь, пользуясь этой таблицей, находим, что

$$
\begin{aligned}
& \varphi(1101)=A\left(b_{2} b_{1}\right)^{l}\left(b_{2} b_{1}\right)^{k} b_{2} b_{2} b_{1} b_{2}\left(b_{1} b_{2}\right)^{t} B=Q_{1}, \\
& \varphi(1100)=A\left(b_{2} b_{1}\right)^{l} b_{2} b_{1}\left(b_{1} b_{2}\right)^{k} b_{2} b_{1}\left(b_{2} b_{1}\right)^{t} B=Q_{2}, \\
& \varphi(1110)=A\left(b_{2} b_{1}\right)^{l}\left(b_{2} b_{1}\right)^{k} b_{2} b_{2} b_{2} b_{1}\left(b_{2} b_{1}\right)^{t} B=Q_{3} .
\end{aligned}
$$

Так как

$$
1101,1100,1110 \in \varepsilon(11010),
$$

должны выполняться соотношения

$$
\varphi(11010) \varepsilon Q_{i}, \quad i=1,2,3 .
$$

Однако нетрудно заметить, что не существует слова $x \in \Omega_{1}^{*}$, удовлетворяющего условию

$$
x \varepsilon Q_{i}, \quad i=1,2,3 .
$$

Полученное противоречие доказывает, что $s \leqslant l$. Из симметричных соображений получим, что $l \leqslant s$ и, значит, $s=l$.

В итоге для слов длины 2 имеем таблицу:

\begin{tabular}{|c|c|}
\hline$P$ & $\varphi(P)$ \\
\hline 00 & $A\left(b_{1} b_{2}\right)^{s} b_{1} b_{1}\left(b_{2} b_{1}\right)^{t} B$ \\
01 & $A\left(b_{1} b_{2}\right)^{s} b_{1} b_{2}\left(b_{1} b_{2}\right)^{t} B$ \\
10 & $A\left(b_{2} b_{1}\right)^{s} b_{2} b_{1}\left(b_{2} b_{1}\right)^{t} B$ \\
11 & $A\left(b_{2} b_{1}\right)^{s} b_{2} b_{2}\left(b_{1} b_{2}\right)^{t} B$ \\
\hline
\end{tabular}

Из этой таблицы видно, что для любого $P=a_{1} a_{2} \in \Omega^{2}$ имеет место формула

$$
\varphi\left(a_{1} a_{2}\right)=A\left(\left(a_{1} \bar{a}_{1}\right)^{s} a_{1} a_{2}\left(\bar{a}_{2} a_{2}\right)^{t}\right)^{\sigma} B
$$

где $\sigma(0)=b_{1}, \sigma(1)=b_{2}$.

Теперь индукцией по $d(P)$ докажем, что в рассматриваемом случае для любого слова $P=a_{1} a_{2} \ldots a_{n} \in \Omega^{*}, n \geqslant 1$, справедлива формула

$$
\varphi\left(a_{1} a_{2} \ldots a_{n}\right)=A\left(\left(a_{1} \bar{a}_{1}\right)^{s} a_{1} a_{2} \ldots a_{n}\left(\bar{a}_{n} a_{n}\right)^{t}\right)^{\sigma} B .
$$

Для $d(P)=2$ формула (31) совпадает с формулой (30) и, значит, доказана. Индуктивный переход от $n$ к $n+1$ осуществляется по той же схеме, что и при доказательстве теоремы 1 , и его детализация здесь не приводится.

Для пустого слова $\Lambda$ образ $\varphi(\Lambda)$ определяется из условий $0 \varepsilon \Lambda, 1 \varepsilon \Lambda$ в двух вариантах (согласно лемме 2):

$$
\varphi(\Lambda)=A\left(b_{1} b_{2}\right)^{s+t} B, \quad \varphi(\Lambda)=A\left(b_{2} b_{1}\right)^{s+t} B
$$

и оба они возможны, поскольку слово $\varphi(\Lambda)$ нигде в доказательстве не использовалось.

Заметим, что найденный класс отображений из $G\left(\Omega, \Omega_{1}, \varepsilon\right)$ охватывается множеством преобразований из теоремы 2.

Теперь рассмотрим случай (b):

$$
\varphi(010)=A b_{2}\left(b_{1} b_{2}\right)^{r+1} B, \quad \varphi(101)=A b_{1}\left(b_{2} b_{1}\right)^{r+1} B .
$$


Здесь слово $\varphi(11)$ удавлетворяет условию

$$
\varphi(101)=A \underline{b_{1}\left(b_{2} b_{1}\right)^{r+1}} B \varepsilon \varphi(11) \varepsilon A b_{2}\left(b_{1} b_{2}\right)^{r} B=\varphi(1) .
$$

Если при переходе от слова $\varphi(101)$ к $\varphi(11)$ удалить первую или последнюю букву подчеркнутого слова, то получится противоречие с инъективностью $\varphi$.

Если удалить внутреннюю букву подчеркнутого слова, то получаем противоречие с условием $\varphi(11) \varepsilon \varphi(1)$.

Если удалить букву из начального подслова $A$, то при переходе от $\varphi(11)$ к $\varphi(1)$ необходимо будет удалить букву из подслова $B$. В этом случае

$$
A=A_{1} c A_{2}, \quad B=B_{1} d B_{2}, \quad A_{1} A_{2} b_{1}=A c A_{2}, \quad b_{1} B_{1} B_{2}=B_{1} d B_{2} .
$$

Отсюда находим, что

$$
c_{1}=b_{1}, \quad A_{2}=b_{1}^{k}, \quad d=b_{1}, \quad B_{1}=b_{1}^{l}
$$

и

$$
\varphi(11)=A\left(b_{2} b_{1}\right)^{r+1} B \text { или } \varphi(11)=A\left(b_{1} b_{2}\right)^{r+1} B .
$$

Получили противоречие с инъективностью $\varphi$.

Таким образом, случай II(b) невозможен.

III. Элементы 0,1 удовлетворяют условию $4^{\circ}$. Пусть

$$
\begin{array}{rlrl}
\varphi(0) & =A b_{1}\left(b_{2} b_{1}\right)^{r} B, & \varphi(1) & =A b_{2}\left(b_{1} b_{2}\right)^{r} B, \quad r>0, \\
\varphi(01) & =A\left(b_{2} b_{1}\right)^{r+1} B, \quad \varphi(10)=A\left(b_{1} b_{2}\right)^{r+1} B .
\end{array}
$$

Здесь, как и в случае II, логически возможны два подслучая

(a) $\varphi(010)=A b_{1}\left(b_{2} b_{1}\right)^{r+1} B, \varphi(101)=A b_{2}\left(b_{1} b_{2}\right)^{r+1} B$,

(b) $\varphi(010)=A b_{2}\left(b_{1} b_{2}\right)^{r+1} B, \varphi(101)=A b_{1}\left(b_{2} b_{1}\right)^{r+1} B$.

Случай III(b) в части отыскания $\varphi(11)$ получается из II(b) заменой $b_{1}$ на $b_{2}$ и $b_{2}$ на $b_{1}$. Поэтому он также приводится к противоречию.

Рассмотрим случай III(a). Здесь $\varphi(11)$ находится из условия

$$
\varphi(101)=A b_{2}\left(b_{1} b_{2}\right)^{r+1} B \varepsilon \varphi(11) \varepsilon A b_{2}\left(b_{1} b_{2}\right)^{r} B=\varphi(1),
$$

полностью совпадающего с условием (27).

Поэтому, как и в случае II(a),

$$
\varphi(11)=A b_{2}\left(b_{1} b_{2}\right)^{s} b_{2}\left(b_{1} b_{2}\right)^{t} B, \quad \varphi(00)=A b_{1}\left(b_{2} b_{1}\right)^{s} b_{1}\left(b_{2} b_{1}\right)^{t} B,
$$

где $s+t>0$. Отсюда и из (32) видно, что здесь для слов $P=a_{1} a_{2}$ длины 2 имеет место формула $\varphi\left(a_{1} a_{2}\right)=A\left(\mu\left(\left(a_{1} \bar{a}_{1}\right)^{s} a_{1} a_{2}\left(\bar{a}_{2} a_{2}\right)^{t}\right)\right)^{\sigma} B$. Теперь индукцией по длине слова доказывается и общая формула $\varphi\left(a_{1} a_{2} \ldots a_{n}\right)=A\left(\mu\left(\left(a_{1} \bar{a}_{1}\right)^{s} a_{1} a_{2} \ldots a_{n}\left(\bar{a}_{n} a_{n}\right)^{t}\right)\right)^{\sigma} B$.

$\mathrm{B}$ итоге получили еще один класс отображений из теоремы 2. Остальные классы аналогичным образом получаются при рассмотрении случаев, когда элементы 0,1 удовлетворяют условию $5^{\circ}$ или $6^{\circ}$.

\section{Список литературы}

1. Бабаш А. В., Глухов М. М., Шанкин Г. П. О преобразованиях множества слов в конечном алфавите, не размножающих искажений. Дискретная математика (1997) 9, №3, 3-19. 